\title{
Women achieve peak freestyle swim speed at earlier ages than men
}

This article was published in the following Dove Press journal:

Open Access Journal of Sports Medicine

9 November 2012

Number of times this article has been viewed

\section{Christoph Alexander Rüst ${ }^{\prime}$ \\ Beat Knechtle 1,2 \\ Thomas Rosemann' \\ 'Institute of General Practice and for Health Services Research, University of Zurich, Zurich, Switzerland; ${ }^{2}$ Gesundheitszentrum St Gallen, St Gallen, Switzerland}

Background: The age of peak swim performance has been investigated for freestyle swimmers for distances ranging from $50 \mathrm{~m}$ to $1500 \mathrm{~m}$ among swimmers aged 19 to 99 years. However, studies have yet to investigate the 10 to 19 year-old age group. The aims of the present study were (1) to investigate the age range of peak freestyle swim speed, and (2) to find differences in age range and peak freestyle swim speed between male and female freestyle swimmers from $50 \mathrm{~m}$ to $1500 \mathrm{~m}$ at a national level.

Methods: The changes in age range and peak freestyle swim speed among Swiss elite freestyle swimmers aged 0-9 years and 70-79 years who were ranked on the Swiss high score list between 2006 and 2010 were analyzed using linear regression analyses and analysis of variance.

Results: Men were fastest at ages 22-23 years for $100 \mathrm{~m}$ and $200 \mathrm{~m}$; at ages 24-25 years for $400 \mathrm{~m}$ and $800 \mathrm{~m}$; and at 26-27 years for $50 \mathrm{~m}$ and $1500 \mathrm{~m}$. Women achieved peak freestyle swim speed at ages 20-21 years for all distances with the exception of $800 \mathrm{~m}$. In the $800 \mathrm{~m}$, women were fastest at ages 26-27 years. The difference in peak freestyle swim speed decreased with increasing swim distance from $50 \mathrm{~m}$ to $800 \mathrm{~m}$ (ie, $13.1 \% \pm 1.3 \%$ in $50 \mathrm{~m} ; 13.2 \% \pm 0.9 \%$ in $100 \mathrm{~m} ; 10.8 \% \pm 0.9 \%$ in $200 \mathrm{~m} ; 7.9 \% \pm 1.3 \%$ in $400 \mathrm{~m}$; and $4.2 \% \pm 2.0 \%$ in $800 \mathrm{~m}$ ). For $1500 \mathrm{~m}$, however, the gender difference increased to $6.4 \% \pm 2.3 \%$.

Conclusion: These findings suggest that peak freestyle swim speed is achieved at lower age ranges in women when compared to men at $50 \mathrm{~m}$ to $1500 \mathrm{~m}$, but not at $800 \mathrm{~m}$. The gender difference in peak freestyle swim speed decreased with increasing swim distance from $50 \mathrm{~m}$ to $800 \mathrm{~m}$, but not for $1500 \mathrm{~m}$. These data should be confirmed with swimmers at an international level.

Keywords: gender, performance, endurance, athlete

\section{Introduction}

It is well known that endurance performance decreases when age increases. ${ }^{1-4}$ In endurance performances such as swimming, peak performance has been described between the ages of 35 and 40 years, with a linear decrease in performance until approximately 70 years of age. ${ }^{2}$ Until the age of approximately 70 years, the decrease in endurance performance is parallel in both women and men. ${ }^{1-3}$ After the age of 70 years, the decline in performance occurs exponentially. ${ }^{1-3}$

For swimmers, the age-related performance decline shows differences regarding both the length of the swim distance as well as gender. ${ }^{2,3}$ In Donato et al, the age-related decline in freestyle swimming performance was greater in the $1500 \mathrm{~m}$ freestyle than in the $50 \mathrm{~m}$ freestyle, and was greater in women than in men in $50 \mathrm{~m}^{2}$ In women, the decline in swimming performance became progressively greater from the $50 \mathrm{~m}$ freestyle to the $800 \mathrm{~m}$ freestyle, as well as to the $1500 \mathrm{~m}$ freestyle. In men, however, 
no differences were observed in the age-related performance decline from the $100 \mathrm{~m}$ freestyle to the $1500 \mathrm{~m}$ freestyle. ${ }^{3}$ Donato et al also reported that the rates of decline in swimming performance associated with age were greater in long duration events compared to short duration events, suggesting a relatively smaller loss of anaerobic muscular power with age compared with cardiovascular endurance. ${ }^{2}$

For coaches and athletes, the knowledge of the age of peak performance in an endurance performance might be of higher interest than knowing the time course of the agerelated decline in performance. When coaches and swimmers know the age of peak freestyle swim speed, the career of an elite swimmer can be better planned. The age of peak endurance performance seemed to remain relatively stable across the years when athletes who had competed in different disciplines and over different distances were investigated. ${ }^{5}$ Schulz and Curnow ${ }^{5}$ analyzed data from between 1896 and 1980 of Olympic track and field athletes as well as swimmers between. They presented the age and peak performance data of Olympic gold medal winners and showed that the age of peak performance remained relatively stable. For swimmers, the age at which the gold medal was won remained remarkably consistent between 1896 and 1980. Men won the gold medal in $100 \mathrm{~m}$ freestyle at the age of $21.4 \pm 3.1$ years, and women won the medal at $19.4 \pm 2.9$ years. For the $400 \mathrm{~m}$ freestyle, men won gold at the age of $19.9 \pm 2.2$ years and women at the age of $17.6 \pm 1.9$ years. For longer distances, men were more likely to win a gold medal at the age of $20.3 \pm 3.5$ years in the $1500 \mathrm{~m}$ freestyle, and women at the age of $16.0 \pm 1.4$ years in the $800 \mathrm{~m}$ freestyle. ${ }^{5}$

However, there seem to be differences regarding the relationship between the age of peak performance and the length of endurance performance for different sports disciplines. For running, several studies investigated the age of peak performance for short-distance runners, ${ }^{5}$ middledistance runners, ${ }^{5}$ half-marathoners, ${ }^{6}$ marathoners, ${ }^{5,6}$ and ultra-marathoners. ${ }^{7-9}$ In runners, the age of peak performance seemed to increase with increasing running distance. ${ }^{5-9}$ Also, among triathletes, the age of peak performance is lower in shorter triathlon distances compared to longer distances. ${ }^{10,11}$ For swimmers, however, younger ages were associated with faster swim speeds in longer distances. ${ }^{5}$ In freestyle swimmers, differences in the age of peak performance associated with swim length have been reported. The age of peak performance in the $1500 \mathrm{~m}$ freestyle was achieved at a younger age ( $\sim 18$ years) compared to the peak performance in the $50 \mathrm{~m}$ freestyle, which was achieved at a higher age $(\sim 23$ years $) .^{12}$
The age of peak swim performance seems to be dependent on gender. Schulz and Curnow ${ }^{5}$ showed that women generally achieved peak swim performances at younger ages than men. In freestyle swimming, however, the association of increased age with increasing distance was reversed for female swimmers, where a younger age of peak performance was associated with a longer swimming distance. Tanaka and Seals ${ }^{3}$ reported that men reached their fastest swim times for $1500 \mathrm{~m}$ freestyle between 25 and 40 years of age, whereas women achieved their fastest $1500 \mathrm{~m}$ freestyle times at the age of 30 to 35 years. In contrast, peak performance in $50 \mathrm{~m}$ freestyle was attained at the age of 20 to 30 years in both women and men. Schulz and Curnow ${ }^{5}$ analyzed the data of Olympic swimmers who competed in $100 \mathrm{~m}$ freestyle, $400 \mathrm{~m}$ freestyle, and $1500 \mathrm{~m}$ freestyle for men, and $100 \mathrm{~m}$ freestyle, $400 \mathrm{~m}$ freestyle, and $800 \mathrm{~m}$ freestyle for women. From 1896 to 1980 , the age of peak swim performance was between approximately 21 years for the $100 \mathrm{~m}$ freestyle and approximately 20 years for $1500 \mathrm{~m}$ freestyle in men, and between approximately 19 years for the $100 \mathrm{~m}$ freestyle, and approximately 16 years for $800 \mathrm{~m}$ freestyle for women.

The age of peak swim performance has been investigated for freestyle swimmers across distances from $50 \mathrm{~m}$ to $1500 \mathrm{~m} \cdot{ }^{2,3,5,13}$ These reports showed different results for the age of peak performance, and some of these reports were based on older data, from 1896-1980, ${ }^{5} 1988-1999,{ }^{2} 1991-1995,{ }^{3}$ and 1993-2001. ${ }^{13}$ In addition, these studies missed investigating the age group encompassing 10-19 years. Donato et $\mathrm{al}^{2}$ investigated athletes aged 19 to 85 years, Fairbrother ${ }^{13}$ examined athletes aged 19 to 96 years, and Tanaka and Seals $^{3}$ assessed athletes aged 19 to 99 years. Since Schulz and Curnow ${ }^{5}$ showed that the age of peak performance in females was $\sim 20$ years or younger, the studies of Donato et $\mathrm{al},{ }^{2}$ Fairbrother, ${ }^{13}$ and Tanaka and Seals ${ }^{3}$ likely failed to determine the correct age of peak swim performance since they excluded swimmers between the ages of 10 and 19 years old from their analyses. Furthermore, Schulz and Curnow ${ }^{5}$ did not investigate all swim distances in their analysis, such as the $200 \mathrm{~m}$ freestyle among women and men, the $1500 \mathrm{~m}$ freestyle among women, and the $800 \mathrm{~m}$ freestyle among men.

Therefore, the aims of the present study were (1) to investigate the age range of peak freestyle swim speed, and (2) to find potential differences in both the age range and the speed of peak freestyle swim performance in male and female top freestyle swimmers for distances from $50 \mathrm{~m}$ to $1500 \mathrm{~m}$. We hypothesized that (1) the age range of peak freestyle swim speed would be lower in women compared to men, and that (2) the age range of peak freestyle swim speed 
would decrease in women with increasing swim distance. To test these hypotheses, we investigated swimmers in the age groups of 0-9 years to 70-79 years who were ranked on the Swiss swimming high score list between 2006 and 2010.

\section{Materials and methods}

All male and female freestyle swimmers who were ranked on the Swiss swimming high score list between 2006 and 2010 were analyzed for their freestyle swim performance on $50 \mathrm{~m}$, $100 \mathrm{~m}, 200 \mathrm{~m}, 400 \mathrm{~m}, 800 \mathrm{~m}$, and $1500 \mathrm{~m}$ distances. The study was approved by the Institutional Review Board of St Gallen, Switzerland, while waiving the requirement for informed consent since the study involved the analysis of publicly available data. The data set from this study was obtained from the Swiss Swimming Federation's website. ${ }^{14}$ The high score list of the Swiss Swimming Federation registers the fastest swim time of each swimmer across all ages for each year. ${ }^{14}$ No swimmer is listed more than twice on the annual high score list.

In total, data were available from 50,519 swimmers, including 24,656 women and 25,863 men who competed in races in $50 \mathrm{~m}$ swimming pools. In order to facilitate data analysis and to make the results more comparable, all swimming times were transformed to swim speed in meters per second by calculating [swim distance $(\mathrm{m})] /[$ swim time (seconds)] prior to statistical analysis. Due to the high variability of performance over time, and given the low number of swimmers per age group in the years preceding 2006, we decided to analyze only swimmers in the five consecutive years between 2006 and 2010.

For the analysis of swim performance across age groups, all swimmers were separated by gender and were further categorized into the following age groups: 0-9 years, 10-19 years, 20-29 years, 30-39 years, 40-49 years, 50-59 years, 60-69 years, and 70-79 years. For each age group and gender, the top three performances (ie, the three fastest swim speeds) per swim distance and age were determined.

The change in age group and swim speed among the top three swimmers was investigated across the 5 years between 2006 and 2010. A one-way analysis of variance showed no significant difference in swim speed for the top three swimmers within the fastest age groups between 2006 and 2010; therefore, the data were pooled together $(\mathrm{n}=3$ subjects $\times 5$ years $=15$ for each age group). In case less than three swimmers were listed in a given age group, that age group was excluded from further data analysis. Data were then analyzed between age groups. The age groups showing the fastest swim speeds were 10-19 years and 20-29 years. In a second step, all swimmers aged 10-29 years were separately analyzed in 2-year age groups (ie, 10-11 years, 12-13 years, 14-15 years, and so on). For each of these age groups we determined the top three swimmers per gender and swim distance and compared swim speeds between age groups.

\section{Statistical analysis}

In order to increase the reliability of the data analyses, each set of data was tested for normal distribution as well as for homogeneity of variances in advance of the statistical analyses. Normal distribution was tested using the D'Agostino-Pearson omnibus normality test, and homogeneity of variances was tested using either Levene's test in case of two groups, and with Bartlett's test in case of more than two groups. To find the significant differences between groups, analysis of variance with subsequent Tukey-Kramer post hoc analysis was employed. Statistical analyses were performed using the Statistical Package for the Social Sciences statistics software ([SPSS] version 19; IBM Corporation, Armonk, NY) and GraphPad Prism (version 5; GraphPad Software, La Jolla, CA). Significance was accepted at $P<0.05$ (two-sided for $t$-tests). Data in the text are given as mean \pm standard deviation (SD).

\section{Results}

For men, peak freestyle swim speed was achieved in the age groups 10-19 years and 20-29 years for all distances (Figure 1). For women, peak freestyle swim speed was achieved in the age groups 10-19 years, 20-29 years, and 30-39 years for the $50 \mathrm{~m}$ freestyle and $400 \mathrm{~m}$ freestyle (Figure 2). For the $100 \mathrm{~m}$ freestyle, $200 \mathrm{~m}$ freestyle, $800 \mathrm{~m}$ freestyle, and $1500 \mathrm{~m}$ freestyle, peak freestyle swim speed was attained in the age groups 10-29 years and 20-29 years.

When the age groups 10-19 years and 20-29 years were analyzed in 2-years intervals, men were fastest between 22-23 years for the $100 \mathrm{~m}$ and $200 \mathrm{~m}$ freestyle, between 24-25 years for the $400 \mathrm{~m}$ and $800 \mathrm{~m}$ freestyle, and between 26-27 years for the $50 \mathrm{~m}$ and $1500 \mathrm{~m}$ freestyle (Figure 3 ). Women achieved their peak freestyle swim speed at the age range of 20-21 years for all distances with the exception of the $800 \mathrm{~m}$ freestyle. In the $800 \mathrm{~m}$ freestyle, women were fastest at 26-27 years (Figure 4).

The difference in peak freestyle swim speed decreased with increasing swim distance from the $50 \mathrm{~m}$ freestyle to the $800 \mathrm{~m}$ freestyle. The gender difference in performance was $13.1 \% \pm 1.3 \%$ for the $50 \mathrm{~m}$ freestyle, $13.2 \% \pm 0.9 \%$ for the $100 \mathrm{~m}$ freestyle, $10.8 \% \pm 0.9 \%$ for the $200 \mathrm{~m}$ freestyle, $7.9 \% \pm 1.3 \%$ for the $400 \mathrm{~m}$ freestyle, and $4.2 \% \pm 2.0 \%$ for 
A

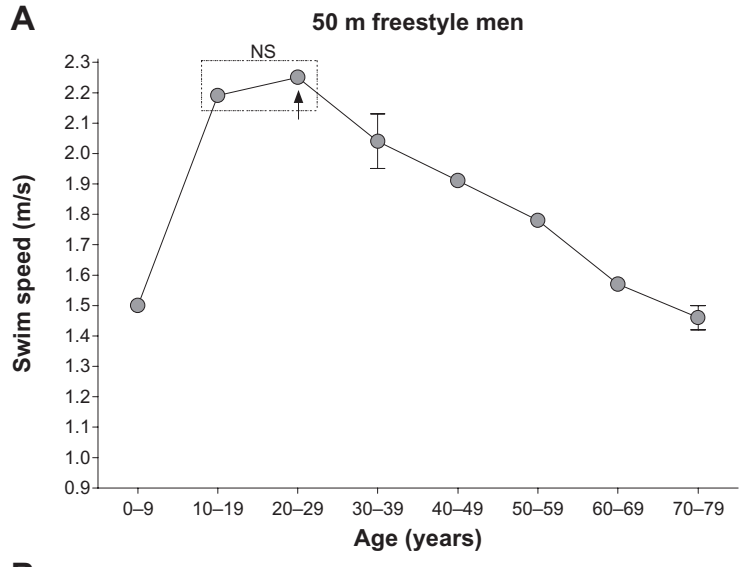

B

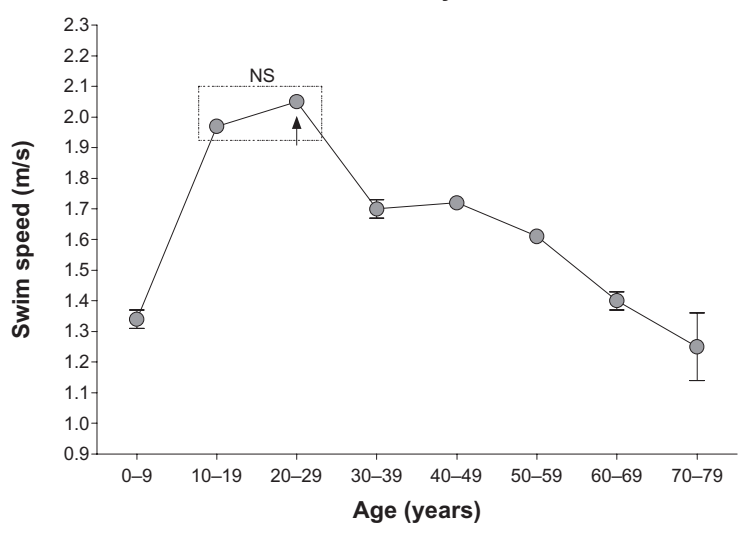

C

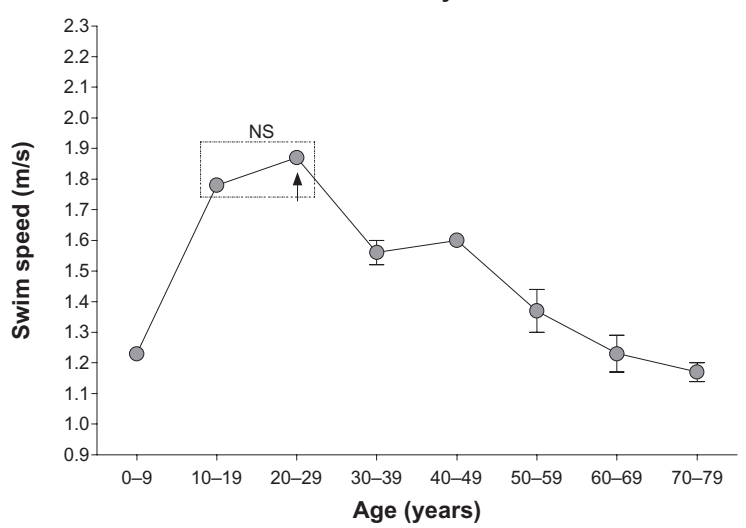

D
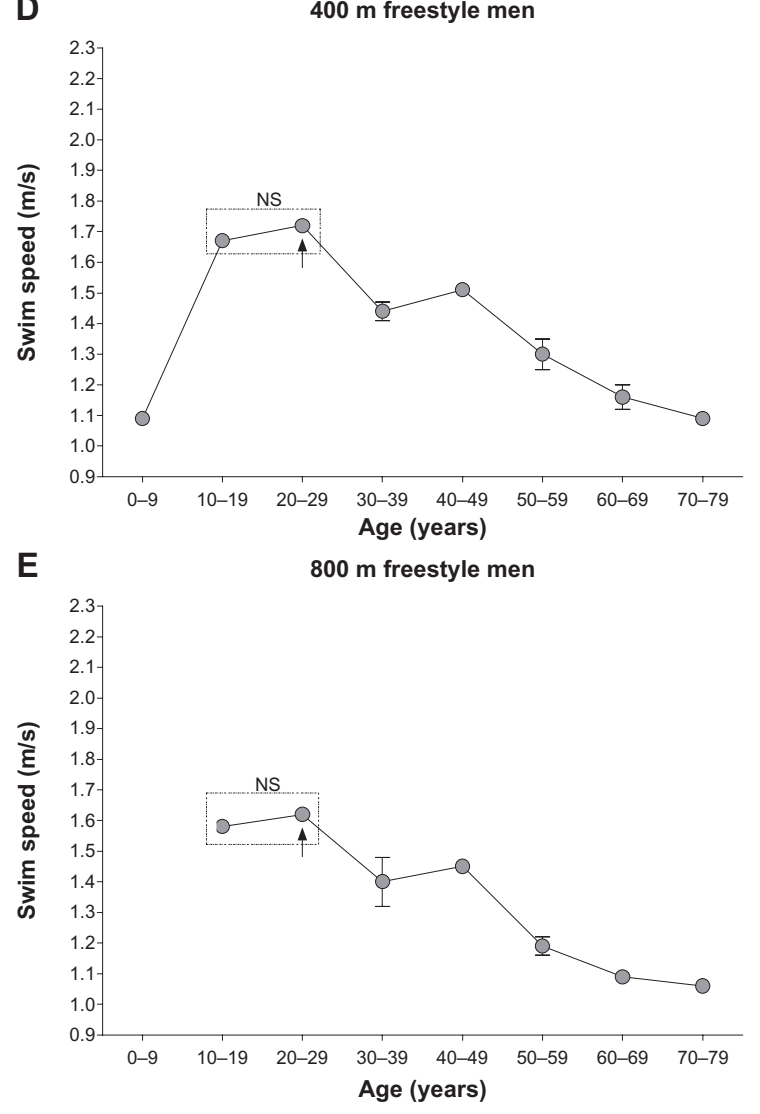

F

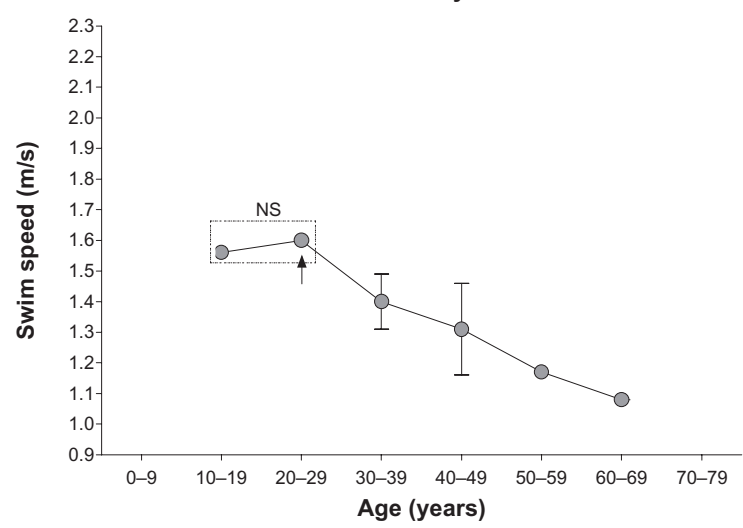

Figure I Swim speed of the three fastest male swimmers in freestyle swimming between 2006 and 2010 in each 10 -year age group based on distance. Swim speed of the three fastest male swimmers in freestyle swimming between 2006 and 2010 in each 10-year age group based on distance for the $50 \mathrm{~m}$ freestyle (A), $100 \mathrm{~m}$ freestyle (B), $200 \mathrm{~m}$ freestyle (C), $400 \mathrm{~m}$ freestyle (D), $800 \mathrm{~m}$ freestyle (E), and $1500 \mathrm{~m}$ freestyle (F).

Note: Age groups with no significant difference in swim speed are indicated by a rectangle and marked with "NS."

the $800 \mathrm{~m}$ freestyle. For the $1500 \mathrm{~m}$ freestyle, however, the difference in peak swim speed increased to $6.4 \% \pm 2.3 \%$.

\section{Discussion}

We intended, firstly, to investigate the age range of peak swim speed in both male and female top swimmers for freestyle distances ranging from $50 \mathrm{~m}$ to $1500 \mathrm{~m}$. Secondly, we also wanted to find potential differences in both the age range and speed of peak freestyle swim performance. With respect to the existing literature investigating the age-related performance decline in freestyle swimming, we hypothesized that (1) the age range of peak freestyle swim speed would be lower in women compared to men, and (2) the age range of peak freestyle swim speed would decrease in women with increasing length of the swim distance. The main findings were that (1) women achieved their peak freestyle swim speed at lower 
A

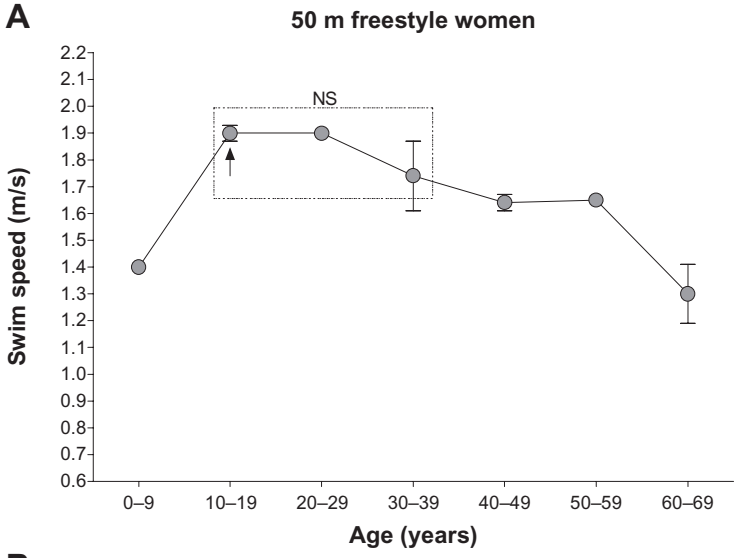

B

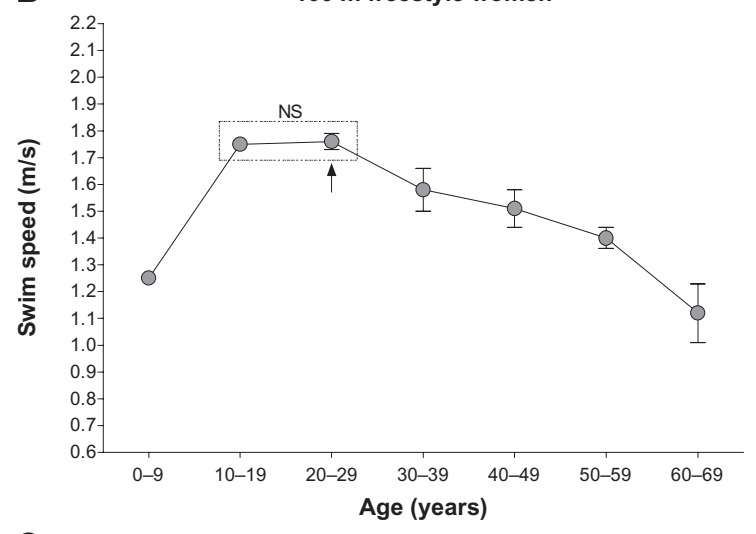

C

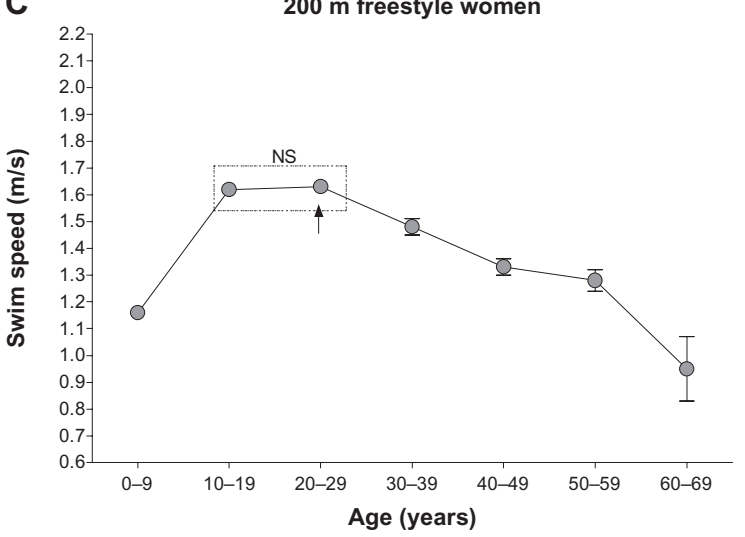

D

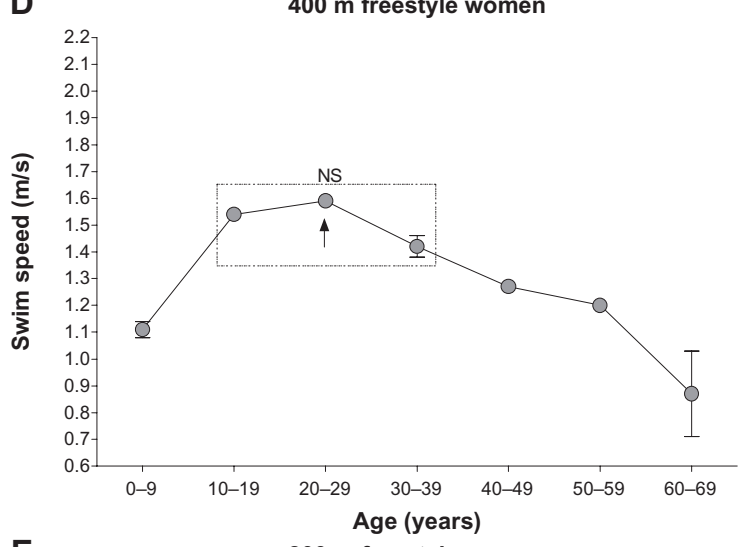

E

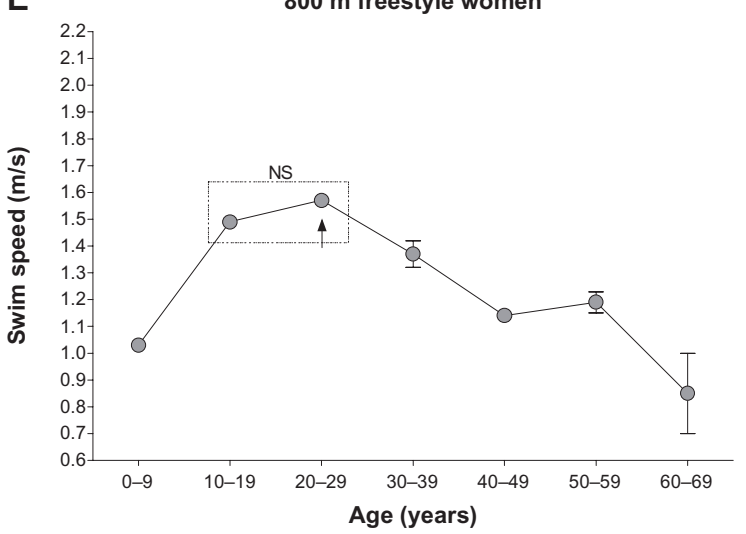

$\mathbf{F}$

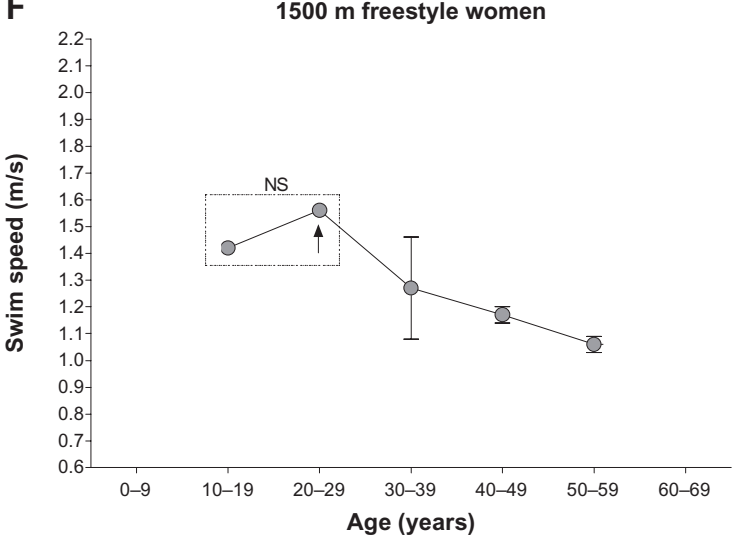

Figure 2 Swim speed of the three fastest male swimmers in freestyle swimming between 2006 and 2010 per 2 -year age group and distance. Swim speed of the three fastest male swimmers in freestyle swimming between 2006 and 2010 per 2-year age group and distance for swimmers aged between 10 and 29 years for the $50 \mathrm{~m}$ freestyle (A), $100 \mathrm{~m}$ freestyle (B), $200 \mathrm{~m}$ freestyle (C), $400 \mathrm{~m}$ freestyle (D), $800 \mathrm{~m}$ freestyle (E), and $1500 \mathrm{~m}$ freestyle (F).

Note: Age groups with no significant difference in swim speed are indicated by a rectangle and marked with "NS."

age ranges compared to men, with the exception of the $800 \mathrm{~m}$ distance, and (2) the gender difference in peak freestyle swim speed decreased with increasing length of the swim distance from $50 \mathrm{~m}$ to $800 \mathrm{~m}$, but not for $1500 \mathrm{~m}$.

\section{Women achieve peak freestyle swim speed at earlier ages than men}

The most important finding was that the age range associated with peak freestyle swim speed was approximately
2 years lower in women compared to men. While Schulz and Curnow $^{5}$ reported that the age range of peak freestyle swim speed was approximately 21 years for men and approximately 19 years for women, we found a considerably higher age range of peak freestyle swim speed for both women and men. The present data differ from the findings of Schulz and Curnow, ${ }^{5}$ who analyzed data of Olympic swimmers from between 1896 and 1980 by investigating the $100 \mathrm{~m}$ freestyle, $400 \mathrm{~m}$ freestyle, and $1500 \mathrm{~m}$ freestyle for men, and the $100 \mathrm{~m}$ 

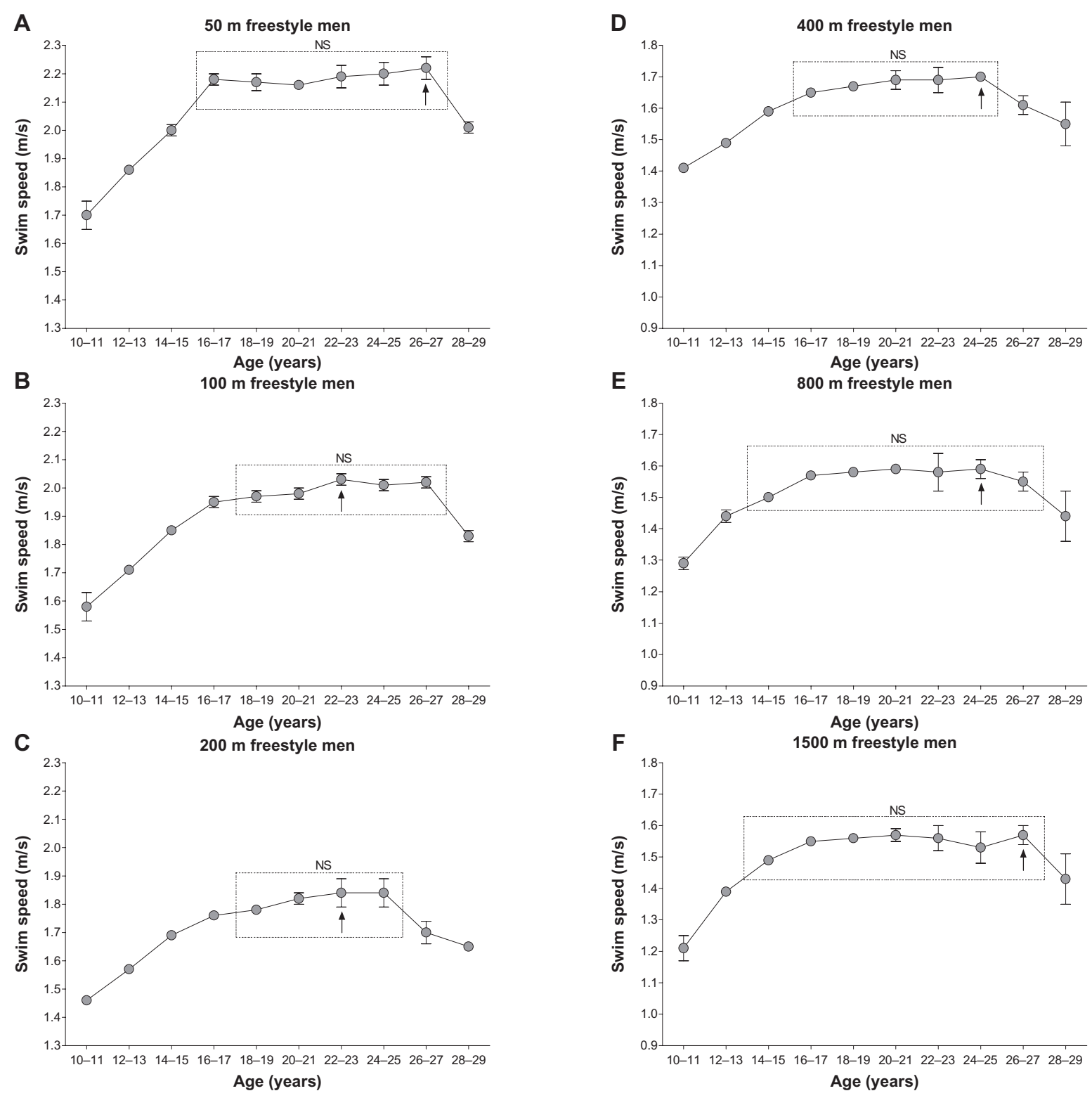

Figure $3 \mathrm{Swim}$ speed of the three fastest female swimmers in freestyle swimming between 2006 and 2010 per 10-year age group and distance. Swim speed of the three fastest female swimmers in freestyle swimming between 2006 and 2010 per 10-year age group and distance for the $50 \mathrm{~m}$ freestyle (A), $100 \mathrm{~m}$ freestyle (B), $200 \mathrm{~m}$ freestyle (C), $400 \mathrm{~m}$ freestyle (D), $800 \mathrm{~m}$ freestyle (E), and $1500 \mathrm{~m}$ freestyle (F).

Note: Age groups with no significant difference in swim speed are indicated by a rectangle and marked with "NS."

freestyle, $400 \mathrm{~m}$ freestyle, and $800 \mathrm{~m}$ freestyle for women. From 1896 to 1980, the age of peak freestyle performance among men was between 21.4 years for the $100 \mathrm{~m}$ freestyle and 20.3 years for the $1500 \mathrm{~m}$ freestyle, while for women it was between 19.4 years for the $100 \mathrm{~m}$ freestyle and 16 years for the $800 \mathrm{~m}$ freestyle. ${ }^{5}$

Schulz and Curnow ${ }^{5}$ separated their data into two halves (ie, 1896-1936 and 1942-1980) using World War II as a dividing point. For men, the age of peak freestyle performance decreased in the $100 \mathrm{~m}$ freestyle from 22.0 years to 20.78 years, in the $400 \mathrm{~m}$ freestyle from 20.44 years to
19.44 years, and in the $1500 \mathrm{~m}$ freestyle from 21.09 years to 19.44 years between the two halves. For women, however, the age of peak freestyle performance increased in the $100 \mathrm{~m}$ freestyle from 18 years to 20.33 years, but the age of peak performance decreased in the $400 \mathrm{~m}$ freestyle from 18 years to 17.33 years. For the $800 \mathrm{~m}$ freestyle, there were no data available from 1986-1936 to compare with the second half after World War 2.

The different findings between the results of Schulz and Curnow $^{5}$ and the present data might be explained by the different time frames of the investigation period and the changes 
A

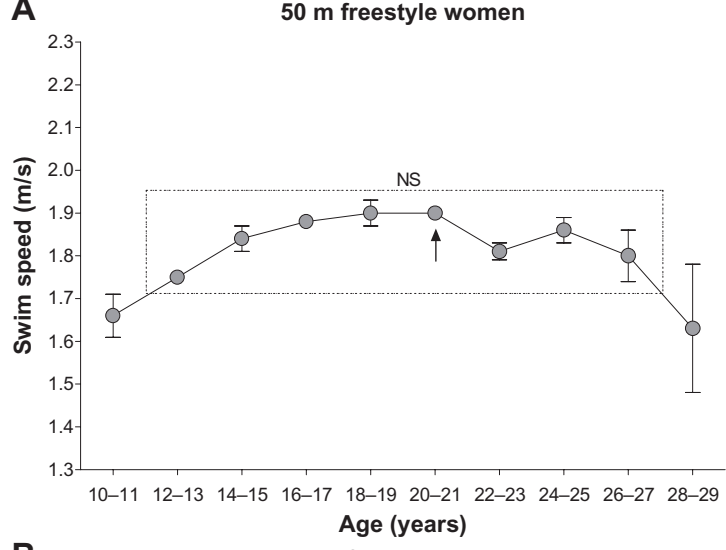

B

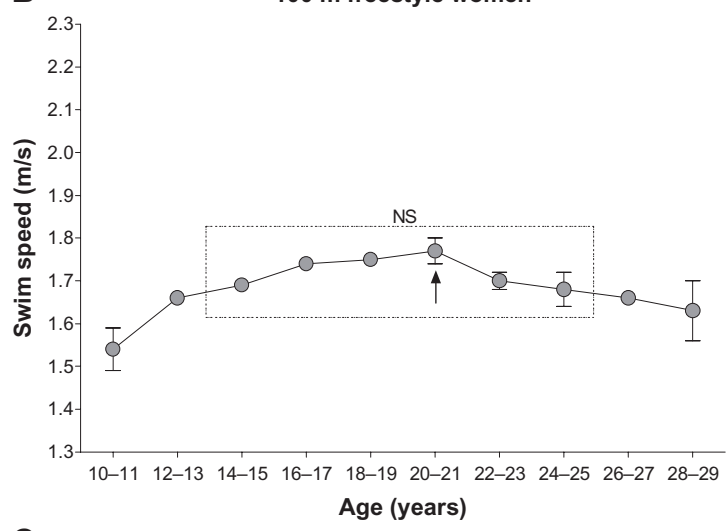

C

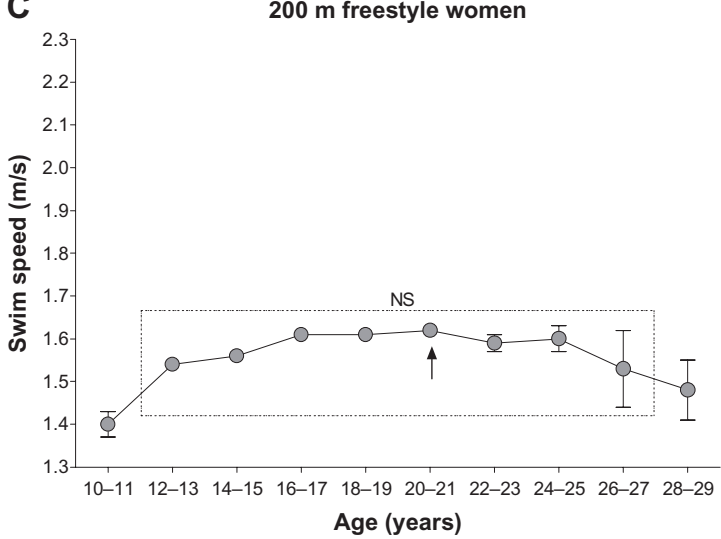

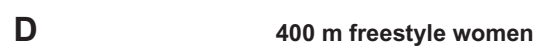

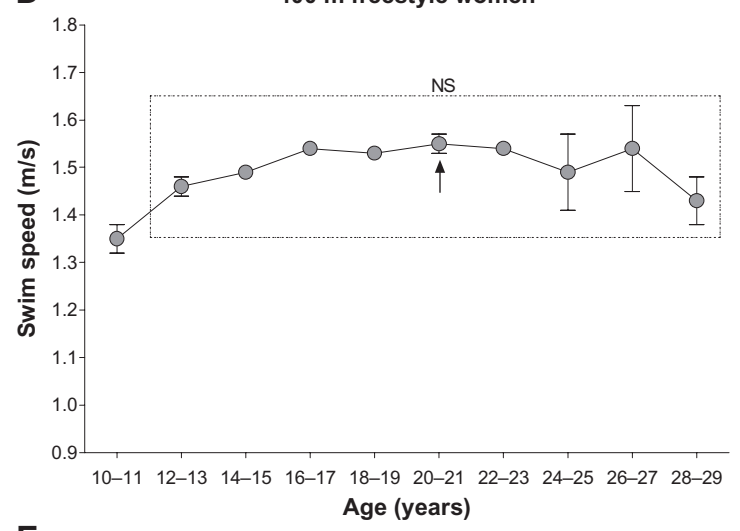

E

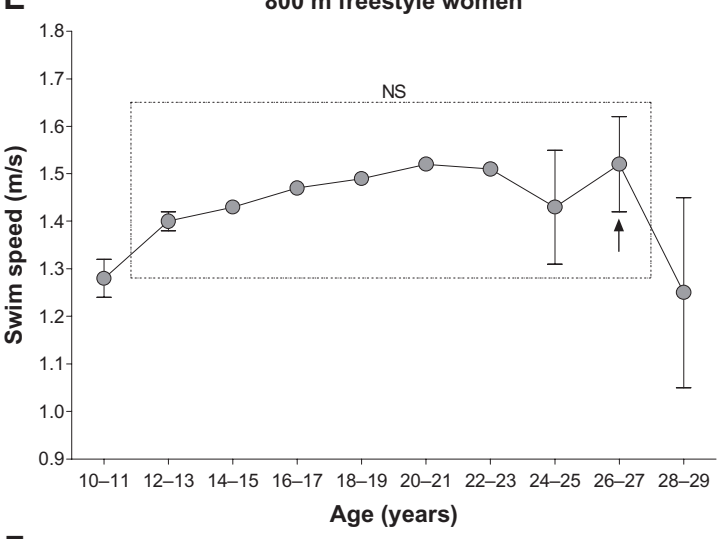

$\mathbf{F}$

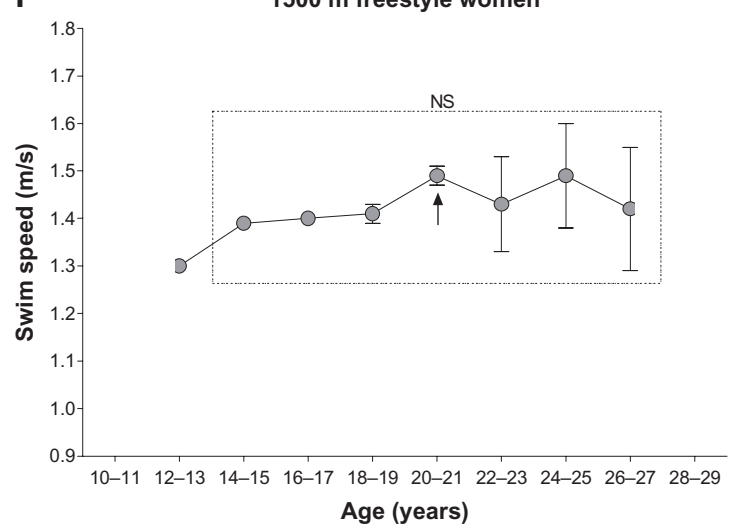

Figure 4 Swim speed of the three fastest female swimmers in freestyle swimming between 2006 and 2010 per 2 -year age group and distance. Swim speed of the three fastest female swimmers in freestyle swimming between 2006 and 2010 per 2-year age group and distance for swimmers aged between 10 and 29 years for the 50 m freestyle (A), $100 \mathrm{~m}$ freestyle (B), $200 \mathrm{~m}$ freestyle (C), $400 \mathrm{~m}$ freestyle (D), $800 \mathrm{~m}$ freestyle (E), and I500 m freestyle (F).

Note: Age groups with no significant difference in swim speed are indicated by a rectangle and marked with "NS."

in anthropometric characteristics across the years. While Schulz and Curnow 5 analyzed data from 1896 to 1980, we analyzed data from 2006 to 2010 . We assume that changes in anthropometric characteristics across the last century might have occurred, explaining these differences. In swimming, anthropometric characteristics such as body height have an influence on performance. ${ }^{15-18}$ Geladas et $\mathrm{a}^{16}$ examined the relationship between anthropometry, physical capacity, and sprint swimming performance in swimmers of both genders aged 12-14 years old. Upper extremity length, horizontal jump, and grip strength were significant predictors of $100 \mathrm{~m}$ freestyle performance in boys. In girls, however, body height, upper extremity and hand length, shoulder flexibility, and horizontal jump were all significantly related to $100 \mathrm{~m}$ freestyle time, but the degree of the association was markedly lower than in boys. Zampagni et al ${ }^{18}$ determined in elite master swimmers of both genders anthropometric predictor variables using anthropometric measures and the hand-grip 
strength measure. Age, height, and hand grip strength were the best predictors in short-distance events, whereas only age and height were predictors in middle- and long-distance events. Differences between sexes were not found in the $50 \mathrm{~m}$ event, but were present in all other events.

In the last several years, anthropometry in adolescents has changed. ${ }^{19-23}$ Kagawa et al ${ }^{19}$ analyzed the changes in growth between 1900 and 2000 in Japanese children from the ages of 6 to 17 years. Over this 100-year period, boys showed increases in height and weight in increments of $1.0-2.0 \mathrm{~cm}$ per decade and $0.4-1.7 \mathrm{~kg}$ per decade, whereas girls demonstrated growth rates of $1.1-1.9 \mathrm{~cm}$ and $0.4-1.5 \mathrm{~kg}$ per decade. The rates of height growth were significantly different between pre-, during, and post-World War II periods. While Japanese children were considerably larger in 2000 compared to 1900, the growth increments noted between 1950 and 1960 reflected children's "catch-up" growth to restore physical size, as seen in children prior to World War II. The increments in body size growth continued after 1960, with the greatest changes seen across the pubertal years. When changes in body dimensions were analyzed for recent decades, Simsek et $\mathrm{al}^{20}$ reported a significant secular increase from 1993 to 2003 in terms of weight and height measurements among 7 to 15-year-old boys and girls in Ankara, Turkey. Also, Marmo et $\mathrm{al}^{21}$ showed that boys and girls exhibited an increase in body height and body weight in São Paulo, Brazil between 1979/1980 and 1993/1994.

Another explanation for the different age ranges associated with peak freestyle swim speed between women and men could include anthropometry and body fat as an important predictor variables for swimming performance. ${ }^{16,24-28}$ Female swimmers have significantly more adipose tissue than male swimmers. ${ }^{24,26,28}$ Siders et $\mathrm{al}^{26}$ reported that measurements of body composition such as body fat and body mass may be predictors of swimming performance in women, but not in men. Tuuri et $\mathrm{al}^{27}$ showed that greater fat mass in female swimmers was more strongly related to lower levels of exercise. While the swimmers achieved their peak freestyle swim speed at approximately 20 years of age, specific changes in body composition during that age period might be of importance. Therefore, the difference in the age of peak freestyle swim speed between genders might be explained by

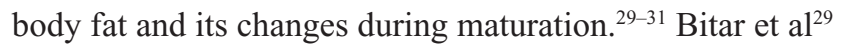
showed that, during the onset of puberty, boys and girls gained fat-free mass, whereas fat mass gain was higher in girls than in boys. Lean body mass, which primarily reflects muscle mass, begins to increase during early puberty in both boys and girls. Fat mass, however, increases during the late stages of puberty in girls. ${ }^{30}$ Further increases in fat mass after puberty may impair swim performance in women. An important finding was reported by Stager et al. ${ }^{31}$ These authors showed that the mean age at menarche in swimmers was significantly later than in controls (ie, 13.4 years and 13 years, respectively). A later menarche means that puberty, as well as its associated increase in fat mass, will also be delayed. Stager et $\mathrm{al}^{31}$ assumed that the later menarche observed in swimmers appeared to be associated with factors that contribute to a superior performance. Zuniga et $\mathrm{al}^{28}$ showed that male and female sprint swimmers at approximately 11 years of age exhibited different percentages of body fat (boys $9.4 \% \pm 5.4 \%$ of body fat; girls $12.7 \% \pm 6.2 \%$ of body fat, respectively), and suggested that swimming performance for girls may be improved through training programs designed to reduce body fat.

Furthermore, maturation occurs earlier in women than in men. ${ }^{30,32}$ This can be seen in bone growth during puberty in relation to physical growth. ${ }^{33}$ Maximal increase of all bone variables, such as width, mineral content, and density, occurred earlier in girls than in boys, with the earliest increase occurring for bone width, mineral content, and density. By the age of 17 years, boys had attained $86 \%$ of the adult bone mineral content and volumetric density, while girls had attained $93 \%$ of the adult bone mineral content and $94 \%$ of volumetric density. A study on the growth of the metacarpal bones showed a difference of approximately 2 years in the growth patterns noted between boys and girls. ${ }^{32}$

\section{Decrease in gender difference with increasing length of swim distance from $50 \mathrm{~m}$ to $800 \mathrm{~m}$}

A second important finding was that the gender difference in peak freestyle swim speed decreased between $50 \mathrm{~m}$ and $800 \mathrm{~m}$. This finding is in line with previous findings observed for the $100 \mathrm{~m}$ to the $400 \mathrm{~m}$ freestyle distances. The analysis of the $100 \mathrm{~m}, 200 \mathrm{~m}$, and $400 \mathrm{~m}$ freestyle swimming world records from 1957 to 2006 showed that gender differences in performance remained stable between $8 \%$ and $11 \% .{ }^{34}$ Regarding the distance of freestyle swimming, the gender difference decreased with increasing swim distance. For the $100 \mathrm{~m}$ freestyle, the gender difference was at $11 \%$; for the $200 \mathrm{~m}$ freestyle, the gender difference was at $10 \%$; and for the $400 \mathrm{~m}$ freestyle, the gender difference was at $9 \% .{ }^{34}$ It seems likely that female swimmers seem to achieve the same performance in longer swim distances as male swimmers. Eichenberger et $\mathrm{al}^{35}$ showed that male and female top swimmers reached the 
same swim distance in a 12 -hour swim in a pool. In the English Channel Swim, the best annual performances did not differ between the sexes. ${ }^{36}$ However, the sex differences in open-water ultra-swimming performance in the $26.4 \mathrm{~km}$ open-water ultra-swim (the Marathon Swim in Lake Zurich, Switzerland), remained unchanged at approximately $11.5 \%$ in the last 25 years. ${ }^{37}$

Anthropometric differences between women and men might explain the differences in the age of the fastest swim speed. Women have proportionally more fatty tissue located caudally compared to men, and the center of buoyancy is different between men and women. ${ }^{24}$ For the longer freestyle distances, the higher body fat in women might play an important role; the higher body fat and the more caudally located body fat may explain why women are able to compete faster over longer distances (ie, in $400 \mathrm{~m}$ freestyle to $1500 \mathrm{~m}$ freestyle) than shorter distances (ie, $50 \mathrm{~m}$ freestyle to $200 \mathrm{~m}$ freestyle). Women seem to have a higher mechanical efficiency compared to men, most probably due to a lower drag. ${ }^{38}$ In addition, body height and the length of the extremities might also explain these differences. Geladas et $\mathrm{al}^{16}$ showed in 2005 that anthropometric characteristics such as extremity length and grip strength were significant predictors of $100 \mathrm{~m}$ freestyle performance in boys. In girls, however, the anthropometric characteristics body height, upper extremity, and hand length were all significantly related to $100 \mathrm{~m}$ freestyle times. Furthermore, body height and arm span were smaller in female swimmers than male swimmers. ${ }^{25}$ Apart from these anthropometric characteristics, differences in stroke frequency and stroke length in freestyle swimming might also explain the present findings. Seifert et $\mathrm{al}^{25}$ analyzed kinematic changes during $100 \mathrm{~m}$ front crawl in both women and men. High-speed swimmers were characterized by a higher and more stable stroke length. In fact, the principal gender difference was the greater stroke length observed in men compared to women.

\section{Greater gender difference in $1500 \mathrm{~m}$ compared to $800 \mathrm{~m}$ freestyle swimming}

We found a decrease in gender difference in peak swim speed between the $50 \mathrm{~m}$ freestyle and $800 \mathrm{~m}$ freestyle; however, for the $1500 \mathrm{~m}$ freestyle, the gender difference increased when compared to performance on the $800 \mathrm{~m}$ freestyle. This finding is in contrast to previous findings. Tanaka and Seals ${ }^{3}$ reported a decrease in gender difference from $50 \mathrm{~m}$ freestyle to $1500 \mathrm{~m}$ freestyle swimming. Also, the authors reported different values for this gender difference. ${ }^{3}$ They showed a gender difference of approximately $20 \%$ for the $50 \mathrm{~m}$ free- style, with a continuous decrease in the gender difference to approximately $10 \%$ for the $1500 \mathrm{~m}$ freestyle.

The most probable explanation for the disparate findings between previous research and the present results in terms of performance in the longer distances such as the $800 \mathrm{~m}$ freestyle and the $1500 \mathrm{~m}$ freestyle is the study population. While Tanaka and Seals ${ }^{3}$ investigated participants between the ages of 19 and 99 years who competed in the US Masters Swimming Championships from 1991-1995, we investigated Swiss elite swimmers between the ages of 0 and 79 years competing between 2006 and 2010. Since the fastest race times were achieved across most distances by swimmers between the ages of 10 and 29 years, the inclusion of the 10-19 age group in the present study may explain the gender differences observed for the longer swim distances.

\section{Limitations}

This study is limited in that anthropometric, ${ }^{26-28,39}$ physiologi$\mathrm{cal}^{40}{ }^{40}$ and training ${ }^{41}$ variables were not included. The athletes' nutrition levels might have also had an influence on their performance. ${ }^{42}$ Variables such as hand grip strength, ${ }^{16,18}$ horizontal jump, ${ }^{16}$ and shoulder flexibility ${ }^{16}$ were not investigated. These variables have been reported to influence swim performance. The inclusion of swimmers aged between 10 and 19 years might have influenced the present findings. Swimmers between 10 and 19 years had undergone puberty; during this period, considerable changes in anthropometric characteristics might have occurred, ${ }^{30}$ which may have affected swim performance. Change in stature has only been investigated with data from the general population, but not with data from athletes. The variability in gains in stature is due to secular trends, heredity, and effects of environmental conditions on the human phenotype, which may have affected swim performance. ${ }^{43,44}$ These aspects were, however, not included in this study. Finally, we compared our data from swimmers between the ages of 0 and 79 years with results from studies where athletes were aged between 19 and 99 years. ${ }^{2,3,5,13}$ The comparison of the data from different age groups with the present findings might also have had an influence on the interpretation of the present data.

\section{Conclusion}

These findings suggest that peak freestyle swim speed is achieved at lower age ranges in women compared to men from the $50 \mathrm{~m}$ freestyle to the $1500 \mathrm{~m}$ freestyle, but not for the $800 \mathrm{~m}$ freestyle. The gender difference in peak freestyle swim speed decreased with increasing length of the swim distance from $50 \mathrm{~m}$ to $800 \mathrm{~m}$, but not for $1500 \mathrm{~m}$. Future 
studies need to investigate differences in age range and peak freestyle swim speed in longer swim distances and in open-water swimming. The present findings should be confirmed with swimmers at an international level.

\section{Disclosure}

The authors report no conflicts of interest in this work.

\section{References}

1. Bongard V, McDermott AY, Dallal GE, Schaefer EJ. Effects of age and gender on physical performance. Age (Dordr). 2007;29(2-3):77-85.

2. Donato AJ, Tench K, Glueck DH, Seals DR, Eskurza I, Tanaka H. Declines in physiological functional capacity with age: a longitudinal study in peak swimming performance. J Appl Physiol. 2003;94(2): 764-769.

3. Tanaka H, Seals DR. Age and gender interactions in physiological functional capacity: insight from swimming performance. J Appl Physiol. 1997;82(3):846-851.

4. Zamparo P. Effects of age and gender on the propelling efficiency of the arm stroke. Eur J Appl Physiol. 2006;97(1):52-58.

5. Schulz R, Curnow C. Peak performance and age among superathletes: track and field, swimming, baseball, tennis, and golf. J Gerontol. 1988; 43(5):P113-P120.

6. Leyk D, Erley O, Ridder D, et al. Age-related changes in marathon and half-marathon performances. Int J Sports Med. 2007;28(6):513-517.

7. Knechtle B, Rüst CA, Rosemann T, Lepers R. Age-related changes in 100-km ultra-marathon running performance. Age (Dordr). 2012;34(4): 1033-1045.

8. Knoth C, Knechtle B, Rüst CA, Rosemann T, Lepers R. Participation and performance trends in multi-stage ultra-marathons - The 'Marathon des Sables' from 2003-2012. Extreme Physiology and Medicine. 2012; In press.

9. Eichenberger E, Knechtle B, Rüst CA, Rosemann T, Lepers R. Age and gender interactions in mountain ultramarathon running - the Swiss Alpine Marathon. Open Access Journal of Sports Medicine. 2012;3:73-80.

10. Knechtle B, Rüst CA, Rosemann T, Lepers R. Age and gender differences in half-Ironman triathlon performances - the Ironman 70.3 Switzerland from 2007 to 2010. Open Access Journal of Sports Medicine. 2012;3:59-66.

11. Knechtle B, Rüst CA, Knechtle P, Rosemann T, Lepers R. Age-related changes in ultra-triathlon performances. Extreme Physiology and Medicine. 2012;1:5.

12. Berthelot G, Len S, Hellard P, et al. Exponential growth combined with exponential decline explains lifetime performance evolution in individual and human species. Age (Dordr). 2012;34(4):1001-1009.

13. Fairbrother JT. Age-related changes in top-ten men's US. Masters $50-\mathrm{m}$ freestyle swim times as a function of finishing place. Percept Mot Skills. 2007;105(3 Pt 2):1289-1293.

14. Highscore list [webpage on the Internet]. Bern; Schweizerischer Schwimmverband. Available from: http://rankings.fsn.ch. Accessed March 24, 2012. German.

15. Avlonitou E. Somatometric variables for preadolescent swimmers. J Sports Med Phys Fitness. 1994;34(2):185-191.

16. Geladas ND, Nassis GP, Pavlicevic S. Somatic and physical traits affecting sprint swimming performance in young swimmers. Int $J$ Sports Med. 2005;26(2):139-144.

17. Jagomägi G, Jürimäe $T$. The influence of anthropometrical and flexibility parameters on the results of breaststroke swimming. Anthropol Anz. 2005;63(2):213-219.

18. Zampagni ML, Casino D, Benelli P, Visani A, Marcacci M, De Vito G. Anthropometric and strength variables to predict freestyle performance times in elite master swimmers. $J$ Strength Cond Res. 2008;22(4): 1298-1307.
19. Kagawa M, Tahara Y, Moji K, Nakao R, Aoyagi K, Hills AP. Secular changes in growth among Japanese children over 100 years (1900-2000). Asia Pac J Clin Nutr. 2011;20(2):180-189.

20. Simsek F, Ulukol B, Gulnar SB. The secular trends in height and weight of Turkish school children during 1993-2003. Child Care Health Dev. 2005;31(4):441-447.

21. Marmo DB, Zambon MP, Morcillo AM, Guimarey LM. Secular trends of growth of schoolchildren from Paulínia, São Paulo-Brazil (1979/80 1993/94). Rev Assoc Med Bras. 2004;50(4):386-390. Portuguese.

22. Kurokawa N, Nakai K, Suzuki K, et al. Trends in growth status among schoolchildren in Sendai, Japan, 1994-2003: leveling-off of mean body height and weight. Tohoku J Exp Med. 2008;216(4):371-375.

23. Krawczynski M, Walkowiak J, Krzyzaniak A. Secular changes in body height and weight in children and adolescents in Poznan, Poland, between 1880 and 2000. Acta Paediatr. 2003;92(3):277-282.

24. McLean SP, Hinrichs RN. Sex differences in the centre of buoyancy location of competitive swimmers. J Sports Sci. 1998;16(4): 373-383.

25. Seifert L, Boulesteix L, Chollet D. Effect of gender on the adaptation of arm coordination in front crawl. Int J Sports Med. 2004;25(3): 217-223.

26. Siders WA, Lukaski HC, Bolonchuk WW. Relationships among swimming performance, body composition and somatotype in competitive collegiate swimmers. J Sports Med Phys Fitness. 1993;33(2):166-171.

27. Tuuri G, Loftin M, Oescher J. Association of swim distance and age with body composition in adult female swimmers. Med Sci Sports Exerc. 2002;34(12):2110-2114.

28. Zuniga J, Housh TJ, Mielke M, et al. Gender comparisons of anthropometric characteristics of young sprint swimmers. $J$ Strength Cond Res. 2011;25(1):103-108.

29. Bitar A, Vernet J, Coudert J, Vermorel M. Longitudinal changes in body composition, physical capacities and energy expenditure in boys and girls during the onset of puberty. Eur J Nutr. 2000;39(4):157-163.

30. Wheeler MD. Physical changes of puberty. Endocrinol Metab Clin North Am. 1991;20(1):1-14.

31. Stager JM, Robertshaw D, Miescher E. Delayed menarche in swimmers in relation to age at onset of training and athletic performance. Med Sci Sports Exerc. 1984;16(6):550-555.

32. Martin DD, Heckmann C, Jenni OG, Ranke MB, Binder G, Thodberg HH. Metacarpal thickness, width, length and medullary diameter in children - reference curves from the First Zürich Longitudinal Study. Osteoporos Int. 2011;22(5):1525-1536.

33. Magarey AM, Boulton TJ, Chatterton BE, Schultz C, Nordin BE, Cockington RA. Bone growth from 11 to 17 years: relationship to growth, gender and changes with pubertal status including timing of menarche. Acta Paediatr. 1999;88(2):139-146.

34. Nevill AM, Whyte GP, Holder RL, Peyrebrune M. Are there limits to swimming world records? Int J Sports Med. 2007;28(12): 1012-1017.

35. Eichenberger E, Knechtle B, Knechtle P, Rüst CA, Rosemann T, Lepers R. No gender difference in peak performance in ultra-endurance swimming performance - Analysis of the 'Zurich 12-h Swim' from 1996 to 2010. Chinese Journal of Physiology. 2012;55:346-351.

36. Eichenberger E, Knechtle B, Knechtle P, Rüst CA, Rosemann T, Lepers R. Best performances by men and women open-water swimmers during the 'English Channel Swim' from 1900 to 2010. J Sports Sci. 2012;30(12):1295-1301.

37. Eichenberger E, Knechtle B, Knechtle P, et al. Sex difference in openwater ultra-swim performance in the longest freshwater lake swim in Europe: Sex difference in ultra-swimming. J Strength Cond Res. Epub June 28, 2012.

38. Pendergast DR, Di Prampero PE, Craig AB Jr, Wilson DR, Rennie DW. Quantitative analysis of the front crawl in men and women. $J$ Appl Physiol. 1977;43(3):475-479.

39. Grimston SK, Hay JG. Relationships among anthropometric and stroking characteristics of college swimmers. Med Sci Sports Exerc. 1986;18(1):60-68. 
40. Pessoa Filho DM, Alves FB, Reis JF, Greco CC, Denadai BS. VO2 kinetics during heavy and severe exercise in swimming. Int $J$ Sports Med. 2012;33(9):744-748.

41. Aspenes ST, Karlsen T. Exercise-training intervention studies in competitive swimming. Sports Med. 2012;42(6):527-543.

42. Rodriguez NR, Di Marco NM, Langley S; for American Dietetic Association, Dietitians of Canada, American College of Sports Medicine. American College of Sports Medicine position stand. Nutrition and athletic performance. Med Sci Sports Exerc. 2009;41(3):709-731.
43. Wells JC, Stock JT. Re-examining heritability: genetics, life history and plasticity. Trends Endocrinol Metab. 2011;22(10):421-428.

44. Wells JC. Maternal capital and the metabolic ghetto: an evolutionary perspective on the transgenerational basis of health inequalities. $\mathrm{Am} \mathrm{J}$ Hum Biol. 2010;22(1):1-17.

Open Access Journal of Sports Medicine

\section{Publish your work in this journal}

Open Access Journal of Sports Medicine is an international, peer-reviewed, open access journal publishing original research, reports, reviews and commentaries on all areas of sports medicine. The manuscript management system is completely online and includes a very quick and fair peer-review system.
Visit http://www.dovepress.com/testimonials.php to read real quotes from published authors. 